\title{
Encoding and retrieval of orientation: A new slant on an old problem
}

\author{
JEAN M. MANDLER \\ University of California, San Diego, La Jolla, California 92093 \\ and \\ NANCY L. STEIN \\ Washington University, Saint Louis, Missouri 63130
}

\begin{abstract}
Two experiments compared various tests of young children's ability to encode and retrieve orientation. A placement test was found to be the most sensitive. With this test, 5-year-old children demonstrate both encoding and retention of diagonal orientation, including its correct direction. Recognition tests and some reconstruction tests resulted in poorer performance. It was suggested that recognition tests provide conflicting perceptual input and reconstruction tests require analysis of part-whole relationships, both of which interfere with the retrieval of orientation.
\end{abstract}

A persistent problem in the study of development is to discover sensitive measures of particular abilities. Whenever we conclude that a child does not have some ability, we are left with the lingering doubt that we did not provide an accurate or sensitive enough measure to test it. Perhaps our test instructions were too complex, or the test overloaded the child with too many choices; the child's failure may be due to the difficulties of the test itself rather than to a lack of the ability being tested.

The present experiments investigated three methods of assessing young children's ability to encode and retrieve orientation. Diagonal orientation was studied because retrieval of diagonal information is a relatively difficult task for young children. However, the general principles discussed should apply broadly to assessment of processing other types of information as well.

It was once thought that young children could not even discriminate certain orientations of geometric forms. Rudel and Teuber (1963) found that children from 5 to 8 years had difficulty in mastering differences in left-right orientation of simple figures in the context of a discrimination learning task. The inappropriateness of using discrimination learning tasks to assess encoding and retention has been previously discussed (Stein \& Mandler, 1974). When a direct perceptual test is used,

This research was supported in part by NIMH Grants MH 24492 and MH 15828. Responsibility for authorship is jointly shared. We would like to thank Bonnie Thompson, who collaborated on Experiment 2, and Marsha Heiman and Becky Glen, who helped in data collection. Special thanks go to the San Diego Unified School District and the Richmond Heights School District in St. Louis for their cooperation. Reprint requests should be sent to Jean M. Mandler, Department of Psychology, C-009, University of California, San Diego, La Jolla, California 92093. for example, a matching-to-sample task, it has been shown that 4-year-olds are capable of discriminating one diagonal orientation from another (Over \& Over, 1967).

When it was discovered that young children can encode diagonal orientation, it was suggested that performance difficulties might be due to failure to retain this information. Indeed, performance on some figures deteriorates when a recognition test involving memory is used, rather than a simultaneous matchingto-sample test (Bryant, 1969). However, assessment of retention is highly dependent on the type of retrieval test used. Performance on recognition tests is a function of the number and similarity of the alternatives provided. Moreover, we have found in pilot studies that 5 -year-old children have more difficulty with a samedifferent recognition test, in which each alternative is presented successively, than with a forced-choice recognition test, in which the same number of alternatives is presented simultaneously. In both cases memory is involved, since the originally presented stimulus is removed. However, in a same-different test the child tends to become confused over successive trials as to which was the original stimulus. In both types of test, performance deteriorates as more choices are offered, even when differences in chance performance are taken into account. These findings suggest that orientation has been coded by the child, but that its retention is fragile. Every presentation of an alternative orientation in a recognition test tends to interfere with the representation of the original orientation. The distractors indeed seem to distract.

An alternative procedure is a reconstruction test. Typically, when children are asked to reconstruct a diagonal from its parts or to reproduce it by drawing, performance is even worse than on a recognition test 
(Olson, 1970; Piaget \& Inhelder, 1956). Both reconstruction from parts or reproduction by drawing require a level of analysis of parts to the whole which is more sophisticated than the mere encoding and storage of orientation per se. It seems reasonable to suppose that reconstruction performance is also a function of the number of parts used. Olson had children reconstruct a diagonal from four or five checkers, which was a difficult task. If the task becomes easier as the number of parts decreases, then a placement test in which the child places a replica of the whole figure in its remembered orientation should be easiest. A one-part placement test is the limit of a reconstruction test and does not require part-whole analysis. Experiment 1 tested this hypothesis. Since a placement task also presents no distracting stimuli, it might also result in better performance than a recognition test. This hypothesis was tested in Experiment 2.

\section{EXPERIMENT 1}

\section{Method}

Subjects and materials. Subjects were 42 children from two kindergarten classes in a lower middle class school in the St. Louis public school system. There were 23 females and 19 males, ranging in age from 5.2 to 6.3 years (mean $=5.8$ ).

Two target pictures were used. Each consisted of a $7.6 \times 7.6-\mathrm{cm}$ white card on which was centered a $5.1 \times 1.3-\mathrm{cm}$ red rectangular bar placed at a $45-\mathrm{deg}$ angle. On one target, the bar was oriented toward the left; on the other, toward the right (45 and $135 \mathrm{deg}$, respectively). Four kinds of test stimuli were used. In each case the subject was given a blank $7.6 \times 7.6-\mathrm{cm}$ white card and one of four versions of the rectangular bar: a whole bar identical to the bar in the target pictures, the same bar cut into two equal parts (each $2.5 \times 1.3 \mathrm{~cm}$ ), three equal parts (each $1.7 \times 1.3 \mathrm{~cm})$, or four equal parts $(1.3 \times 1.3 \mathrm{~cm})$.

Procedure. Subjects were tested individually. First, a pretraining task was given, designed to inform the children that orientation of figures was important to the task. Pretraining consisted of three matching-to-sample problems, in each of which subjects had to pick one of three alternative pictures which exactly matched a target picture of a simple geometric form. Each alternative differed from the target picture only in orientation or location. When subjects had made their choice they were asked to explain how the other alternatives differed from the target. All subjects completed the pretraining task successfully.

Subjects were then divided into four groups. Each group saw one of the two target pictures for $15 \mathrm{sec}$. Next, three of the groups were asked to reconstruct the bar on a blank card. One group $(\mathrm{N}=12)$ was given four parts, one group $(\mathrm{N}=10)$ was given three parts, and the remaining group $(N=10)$, two parts. The fourth group $(\mathrm{N}=10)$ was given a placement task; they were handed a single whole bar and asked to place it in the correct orientation. The interval between viewing the target and beginning the reconstruction or placement test was approximately $20 \mathrm{sec}$, during which time the children counted to 20 aloud.

After a 2- to 3-min break, all children were given a second task. They were shown the second target picture (in the opposite orientation to the first target) for $15 \mathrm{sec}$, and then given the placement task. Half of each group were shown the left diagonal first, half the right diagonal first.

\section{Results}

A bar was considered to be correctly reconstructed or placed if it fell within $22.5 \mathrm{deg}$ on either side of the true diagonal. This criterion allows an equal range of error around the horizontal and vertical coordinates as well as both diagonals. On Task 1, performance improved as the number of parts was decreased $\left(\chi^{2}=9.29, \mathrm{p}<.02\right){ }^{1}$ Thirty-three percent of the subjects in the four-part group reconstructed the correct orientation, $70 \%$ in the three-part group, $80 \%$ in the two-part group, and $90 \%$ of the placement subjects were correct. On Task 2, 90\%-92\% of the subjects in all four groups placed the bar in its correct orientation. There were no significant sex differences in performance.

In the two- and three-part reconstruction conditions, children had no difficulty in aligning the parts. Errors were occasionally made in orientation, but the parts were always correctly connected and, thus, the direction of the resulting reconstruction did not waver. In the four-part condition, only 3 of the 12 reconstructions wavered in the fashion so frequently found by Olson (1970) using checkers and by Piaget and Inhelder (1956) using posts on a table top. In these three cases, the subjects placed the first part in a more or less diagonal orientation and then placed the remaining parts in an increasingly vertical orientation. It might be noted that the parts in this condition consisted of identical squares. It was the most similar to the checkerboard reconstruction of the diagonal extensively studied by Olson, who has documented the difficulty young children have in reconstructing a diagonal from components consisting of a number of identical parts. In his situation, as in our four-part condition, the individual components provide no orientation information relevant to the whole. The parts in the two- and three-part conditions were each small rectangles, in effect miniature versions of the whole bar. Thus, the four-part task not only had more parts, but the parts themselves did not offer the same degree of memorial support as in the other conditions.

Errors in orientation were classified as horizontal, vertical, or opposite diagonal errors. Across the various conditions, $7 \%$ were horizontal errors, $47 \%$ vertical errors, and $47 \%$ opposite diagonal errors. The majority of the vertical errors occurred in the four-part reconstruction condition.

\section{EXPERIMENT 2}

In contrast to previous data, Experiment 1 showed that 5-year-old children are not only able to encode diagonal information but can retrieve that information from memory if a sensitive retrieval test is used. Experiment 2 compared the placement task with the more commonly used recognition task. Neither type of test involves the part-whole analyses required by a reconstruction test, and it is not obvious that one should be easier than the other. A placement test is a recall test; a recognition test includes a copy cue which might be expected to facilitate performance, but it also includes distracting cues. 


\section{Method}

Subjects and materials. Two replications were carried out. One consisted of 120 kindergarten children from three public schools in San Diego, ranging in age from 5.0 to 5.9 years $($ mean $=5.5)$. The other consisted of 120 kindergarten children from two schools in St. Louis, ranging in age from 5.2 to 6.4 years $($ mean $=5.9)$. In each replication, half of the children were black and half were white. Approximately half were male and half female. The SES of both groups was lower middle class. ${ }^{2}$

The target pictures and bars used for placement were the same as in Experiment 1. Although the placement test allowed four alternatives (horizontal, vertical, correct diagonal, and opposite diagonal), it was decided to use only a three-alternative recognition test. Pilot work had shown that a four-alternative test was more difficult than a three-alternative one, and it was desired to make the recognition test as easy as possible. The three alternatives consisted of a replica of the target and two distractors. One distractor contained a vertical bar and the other an opposite diagonal bar. A horizontal distractor was not used because children rarely choose a horizontal bar in recognition tests, although they do occasionally place a bar horizontally in a placement test. It is not known why this difference should obtain between the two types of tests, except that the perceptual context provided by a recognition test seems to emphasize the vertical; a 45-deg diagonal in that context appears more vertical than horizontal.

Procedure. The 120 subjects in each replication were divided into two ethnic groups of 60 each. Each group was divided in half, 30 subjects receiving a recognition test, 30 a placement test. The procedure was similar to that used in Experiment 1. Subjects were given the same pretraining task. Then they were shown a target stimulus for $15 \mathrm{sec}$ and told to remember it. The placement task and instructions were identical to Experiment 1 . The recognition task was a three-alternative forcedchoice task, consisting of the simultaneous presentation of the target and two distractors placed in a row. Subjects were asked to point to the picture that looked exactly the same as the one they had just seen. Left vs right orientation of targets and the position of target and distractors in the recognition test were counterbalanced across subjects.

\section{Results}

There were no significant differences in performance due to sex or ethnic group. Children in the St. Louis sample were slightly older and performed better than those in San Diego $\left(\chi^{2}=4.22, \mathrm{p}<.05\right)$. In spite of the fact that there were more opportunities for error in the placement test, placement was significantly better than recognition, $73 \%$ vs $58 \%$ correct $\left(\chi^{2}=6.64, p<.01\right)$. The recognition test had a $33 \%$ correct guessing rate and the placement test only a $25 \%$ correct guessing rate. Thus, even using a more stringent criterion for the placement test, performance was nevertheless significantly better. When a more liberal scoring of placement performance was used (a range of $30 \mathrm{deg}$ on either side of the diagonals), performance improved only slightly, from $73 \%$ to $76 \%$ correct. Subjects tended to restrict their placements to the horizontal, vertical, and diagonal coordinates. Placement on the horizontal and vertical was quite exact; the mean deviation was $1.9 \mathrm{deg}$ to either side of the vertical and $3.2 \mathrm{deg}$ to either side of the horizontal. The mean deviation for the correct diagonal was $7.5 \mathrm{deg}$, and for the opposite diagonal $8.3 \mathrm{deg}$. In this experiment $21 \%$ of the placement errors were horizontal, $28 \%$ vertical, and $52 \%$ opposite diagcnal errors. On the recognition test, $41 \%$ of the errors were vertical, and $59 \%$ were opposite diagonal errors.

\section{DISCUSSION}

Why should a placement task be a more sensitive measure of retention of orientation than a recognition test? The data suggest that the retention of the orientation of some figures is fragile and easily disrupted. When young children are presented with a series of alternatives in a recognition test, the perceptual input of the various alternatives interferes with the remembered information. When they are not given conflicting perceptual information, but are asked to recreate their representation on a blank sheet, performance markedly improves.

The placement task does not involve the analysis of parts to whole that a reconstruction task requires. In addition to the greater difficulty when part-whole analysis is required, the reconstruction task may also involve perceptual interference. Children showed little difficulty in placing a bar broken into two or three parts. Each of these parts looked more or less like the whole bar, in the sense of retaining rectangular shape. Children given four parts not only had one more part to deal with, but each part was a square and bore no perceptual relationship to the whole; that is, the task broke down an integral figure into undifferentiated units. In this sense, the four-part reconstruction task was the only one of comparable difficulty to the tasks used by Olson (1970) and Piaget and Inhelder (1956). It is suggestive that the greatest proportion of vertical errors occurred in the four-part reconstruction task. Furthermore, it was only in this condition that a constant line of direction was not maintained and tended to veer toward the vertical. Presentation of a bunch of squares may suggest to the child orthogonality or "up and down" and thus the diagonal representation is lost.

In any case, the representation of the diagonal by 5-year-old children includes more precise information than a mere match or mismatch with an orthogonal frame of reference (Bryant, 1974). Direction cannot be accounted for by a match-mismatch code alone, and it is clear that the 5-year-old child is capable of forming a representation of diagonality which includes its correct direction. Enough perceptual analysis has been carried out so that the representation can be used to place a bar correctly or even to maintain reconstruction from parts, as long as the parts bear some meaningful relationship to the whole.

\section{REFERENCES}

Bryant, P. E. Perception and memory of the orientation of visually presented lines by children. Nature, 1969, 224, 1331-1332.

Bryant, P. E. Perception and understanding in young children. London: Methuen, 1974.

Ouson, D. Cognitive development: The child's acquisition of diagonality. New York: Academic Press, 1970.

Over, R., \& Over, J. Detection and recognition of mirrorimage obliques by young children. Journal of Comparative and Physiological Psychology, 1967, 64, 467-470.

Piaget, J., \& Inhelder, B. The child's conception of space. London: Routledge \& Kegan Paul, 1956.

Rudel, R. G., \& Teuber, H. L. Discrimination of direction of line in children. Journal of Comparative and Physiological Psychology, 1963, 56, 892-898.

Stein, N. L., \& Mandler, J. M. Children's recognition of 
reversals of geometric figures. Child Development, 1974. 45, 604-615.

\section{NOTES}

1. Chi-square tests were used rather than analyses of variance on degrees of error in placement because of the tendency for scores to cluster discretely around the true coordinates and diagonals.
2. A large number of subjects were used in this experiment since it formed part of an ongoing project on ethnic differences in perception. In addition, it included various labeling conditions, in which the experimenter or the subjects provided labels for the bars, or no labels were used. Labeling had no effect on performance and will not be further considered.

(Received for publication February 10, 1977.) 